Review

\title{
The burden and etiology of lower respiratory tract infections in children under five years of age in Indonesia
}

\author{
Vincentia Rizke Ciptaningtyas ${ }^{1,2}$, Quirijn de Mast $^{3}$, Marinus Isaäk de Jonge ${ }^{2}$ \\ ${ }^{1}$ Department of Microbiology, Faculty of Medicine, Universitas Diponegoro, Semarang, Indonesia \\ 2 Section Pediatric Infectious Disease, Laboratory of Medical Immunology, Department of Laboratory Medicine, \\ Radboud Center for Infectious Diseases, Radboud Institute for Molecular Life Sciences, Radboud university \\ medical center, Nijmegen, the Netherlands \\ ${ }^{3}$ Department of Internal Medicine, Radboud Center for Infectious Diseases, Radboud university medical center, \\ Nijmegen, The Netherlands
}

\begin{abstract}
Introduction: Lower respiratory tract infections (LRTI) are a substantial threat for children worldwide. Currently, there is a lack of knowledge about the burden and etiology of LRTI in children under five years of age in Indonesia.

Methodology: We have systematically searched the available English and Indonesian scientific literature to review and summarize data on LRTI and LRTI-associated invasive disease, and bacterial carriage in the upper respiratory tract in children under five years of age in Indonesia. Results: Overall, data on the burden and etiology of LRTI in children under five years of age in Indonesia is very limited. The data are primarily collected in Java. Data from other parts of Indonesia, including Sumatra, Kalimantan, and Sulawesi, are scarce. The case fatality rate (CFR) of LRTI in children under five years of age in Indonesia was $0.11 \%$. Influenza was the most commonly reported viral etiological agent of LRTI in children under five years of age in Indonesia. Klebsiella pneumoniae was the most frequently reported bacterial agent of LRTI. Streptococcus pneumoniae showed the highest carriage rates.

Conclusions: Surveillance and diagnostic studies are urgently needed and should be conducted in different parts of Indonesia to improve insight in the burden and etiology of LRTI in Indonesia. These data are pivotal to increase the effectiveness of public health strategies, including vaccination and prevention of antimicrobial resistance.
\end{abstract}

Key words: Children; etiology; Indonesia; lower respiratory tract infection.

J Infect Dev Ctries 2021; 15(5):603-614. doi:10.3855/jidc.14268

(Received 28 October 2020 - Accepted 03 November 2020)

Copyright $(C 2021$ Ciptaningtyas et al. This is an open-access article distributed under the Creative Commons Attribution License, which permits unrestricted use, distribution, and reproduction in any medium, provided the original work is properly cited.

\section{Introduction}

Lower respiratory tract infections (LRTI) are a substantial threat for children resulting in high morbidity and mortality rates worldwide. It is estimated that, globally, $15.5 \%$ of children mortality is due to LRTI [1]. Indonesia is one of the developing countries with a high contribution to global pneumonia cases [2]. Previous studies have been conducted to determine strategies to reduce the burden of LRTI in Indonesia through surveillance and vaccination [3-7]. In several epidemiological studies, carriage rates of potentially pathogenic bacteria, i.e. Streptococcus pneumoniae, Staphylococcus aureus, Haemophilus influenzae, Moraxella catarrhalis and also Klebsiella pneumoniae were measured in healthy children in Central Java, West Java, and West Sumatra provinces [8-10]. The serotype distribution of $S$. pneumoniae was studied in Jakarta and West Nusa Tenggara to determine the effectiveness of the pneumococcal vaccine [11-13]. Although the Ministry of Health of the Republic of Indonesia has tried to investigate the etiology of LRTI in children through Severe Acute Respiratory Infection (SARI) surveillance, currently, many findings have not been published in international journals and are thus not accessible through medical literature databases [14]. In order to obtain insight into what is currently known about the burden of LRTI, associated invasive disease and bacterial carriage in Indonesian children, we systematically searched all available English and Indonesian literature.

\section{Methodology}

The PRISMA checklist was followed for the development of our study protocol [15]. The systematic review protocol of this study was registered in PROSPERO under number CRD42020153212 
(available

at

https://www.crd.york.ac.uk/prospero/display_record.p hp?RecordID=153212).

\section{Eligibility criteria}

Studies on LRTI in children under five years of age in Indonesia were eligible for inclusion. In this paper, the term LRTI is used in its broadest sense referring to all infections in the lungs or below the vocal cord, excluding tuberculosis. Additionally, we included studies that have stratified age data of children below the age of five. Exclusion criteria were studies without age-specific data, studies without any information on pathogen identification, review articles, either systematic or narrative, and posters.

\section{Literature search}

We searched the following electronic databases: PubMed, Embase, Web of Science, Scopus, and ProQuest for studies published between January 2009 and October 2019. The search was limited to publications written in English and Indonesian. We were using a combination of search strings for lower respiratory tract infection, children, and Indonesia. In addition, we used Google Scholar to search for articles written in Indonesian using the keywords pneumonia, and anak (children).

\section{PUBMED}

1. "Respiratory Tract Infections"[Mesh:NoExp] OR "Bronchitis"[Mesh] OR "Empyema, Pleural"[Mesh] OR "Lung Abscess"[Mesh] OR "Lung Diseases, Fungal"[Mesh] OR "Lung Diseases, Parasitic"[Mesh] OR "Pleurisy"[Mesh] OR "Pneumonia"[Mesh] OR Pneumon*[tiab] OR bronchopneumon*[tiab] OR pleuropneumon*[tiab] OR Bronchitis[tiab] OR Bronchiolitis[tiab] OR CAP[tiab] OR HAP[tiab] OR VAP[tiab] OR Lower respiratory tract infection*[tiab] OR LRTI[tiab] OR Lower respiratory infection*[tiab] OR Lung disease*[tiab] OR Lung infection*[tiab] OR Pulmonary disease*[tiab] OR Pulmonary infection*[tiab]

2. child*[tw] OR schoolchild*[tw] OR infan*[tw] OR adolescen*[tw] OR pediatri*[tw] OR paediatr*[tw] OR neonat*[tw] OR boy[tw] OR boys[tw] OR boyhood[tw] OR girl[tw] OR girls[tw] OR girlhood[tw] OR youth[tw] OR youths[tw] OR baby[tw] OR babies[tw] OR toddler*[tw] OR newborn*[tw] OR postneonat*[tw] OR postnat*[tw] OR perinat* $[\mathrm{tw}]$ OR $\operatorname{preschool}^{*}[\mathrm{tw}] \quad \mathrm{OR}$ suckling*[tw]

3. ("Indonesia"[Mesh] OR West Irian*[tiab] OR Indonesia*[tiab] OR Irian Jaya*[tiab] OR Java[tiab] OR Javanes*[tiab] OR Bali[tiab] OR Balines*[tiab] OR Sumatra*[tiab] OR Celebes*[tiab] OR Sulawesi*[tiab] OR Madoera*[tiab] OR Madura*[tiab]

4. \#1 AND \#2 AND \#3

\section{EMBASE}

1. exp pneumonia/ OR lower respiratory tract infection/ or exp chest infection/ or lung infection/ OR Pneumon*.ti,ab,kw. OR bronchopneumon*.ti,ab,kw. OR pleuropneumon*.ti,ab,kw. OR Bronchitis.ti,ab,kw. OR Bronchiolitis.ti,ab,kw. OR CAP.ti,ab,kw. OR HAP.ti,ab,kw. OR VAP.ti,ab,kw. OR Lower respiratory tract infection*.ti,ab,kw. OR LRTI.ti,ab,kw. OR Lower respiratory infection*.ti,ab,kw. OR Lung disease*.ti,ab,kw. OR Lung infection*.ti,ab,kw. OR Pulmonary disease*.ti,ab,kw. OR Pulmonary infection*.ti,ab,kw.

2. babies.ti,ab,kw. OR baby.ti,ab,kw. OR boy.ti,ab,kw. OR boyhood.ti,ab,kw. OR boys.ti,ab,kw. OR Exp child health care/ OR Exp child health/ OR child*.ti,ab,kw. OR Exp child/ OR Exp childhood disease/ OR Exp childhood mortality/ OR Exp childhood/ OR girl.ti,ab,kw. OR girlhood.ti,ab,kw. OR girls.ti,ab,kw. OR Exp high risk infant/ OR infan*.ti,ab,kw. OR Exp infant disease/ OR Exp infant mortality/ OR neonat*.ti,ab,kw. OR Exp newborn disease/ OR Exp newborn morbidity/ OR Exp newborn period/ OR newborn*.ti,ab,kw. OR Exp newborn/ OR nicu.ti,ab,kw. OR paediatr*.ti,ab,kw. OR pediatr*.ti,ab,kw,hw. OR exp pediatric nursing/ OR exp pediatric rehabilitation/ OR exp newborn hypoxia/ OR exp pediatric ward/ OR exp pediatrics/ OR perinat*.ti,ab,kw. OR Exp perinatal development/ OR Exp perinatal period/ OR picu.ti,ab,kw. OR postnat*.ti,ab,kw. OR postneonat*.ti,ab,kw. OR preschool*.ti,ab,kw. OR school child.ti,ab,kw. OR schoolchild*.ti,ab,kw. OR suckling*.ti,ab,kw. OR toddler*.ti,ab,kw. OR youth.ti,ab,kw. OR youths.ti,ab,kw.

3. exp indonesia/ OR West Irian*.ti,ab,kw. OR Indonesia*.ti,ab,kw. OR Irian Jaya*.ti,ab,kw. 
OR Java.ti,ab,kw. OR Javanes*.ti,ab,kw. OR Bali.ti,ab,kw. OR Balines*.ti,ab,kw. OR Sumatra*.ti,ab,kw. OR Celebes*.ti,ab,kw. OR Sulawesi*.ti,ab,kw. OR Madoera*.ti,ab,kw. OR Madura*.ti,ab,kw.

4. \#1 AND \#2 AND \#3

\section{WEB OF SCIENCE (AND SCOPUS)}

1. pneumonia OR chest infection or lung infection OR Pneumon* OR bronchopneumon* OR pleuropneumon* OR Bronchitis OR Bronchiolitis OR CAP OR HAP OR VAP OR Lower respiratory tract infection* OR LRTI OR Lower respiratory infection* OR Lung disease* OR Lung infection* OR Pulmonary disease* OR Pulmonary infection*

2. babies OR baby OR boy* OR child* OR girl* OR infan* OR neonat* OR newborn* OR nicu

Figure 1. Flowchart of study selection.

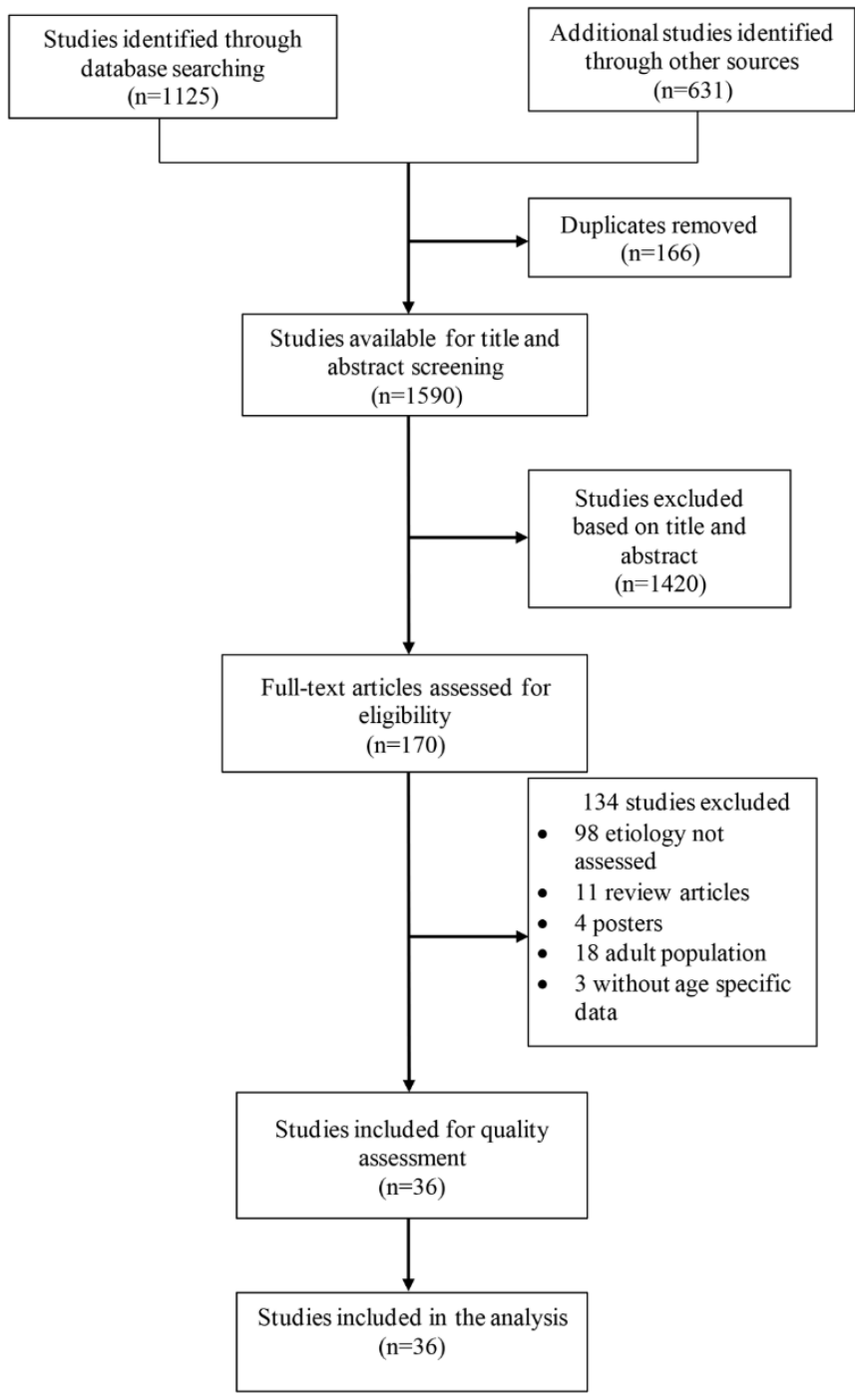

OR paediatr* OR pediatr* OR perinat* OR picu OR postnat* OR postneonat* OR preschool* OR schoolchild* OR suckling* OR toddler* OR youth*

3. West Irian* OR Indonesia* OR Irian Jaya* OR Java OR Javanes* OR Bali OR Balines* OR Sumatra* OR Celebes* OR Sulawesi* OR Madoera* OR Madura*

4. \#1 AND \#2 AND \#3

\section{PROQUEST}

1. pneumonia OR chest infection or lung infection OR Pneumon* OR bronchopneumon* OR pleuropneumon* OR Bronchitis OR Bronchiolitis OR CAP OR HAP OR VAP OR Lower respiratory tract infection* OR LRTI OR Lower respiratory infection* OR Lung disease* OR Lung infection* OR Pulmonary disease* OR Pulmonary infection*

2. babies OR baby OR boy* OR child* OR girl* OR infan* OR neonat* OR newborn* OR nicu OR paediatr* OR pediatr* OR perinat* OR picu OR postnat* OR postneonat* OR preschool* OR schoolchild* OR suckling* OR toddler* OR youth*

3. West Irian* OR Indonesia* OR Irian Jaya* OR Java OR Javanes* OR Bali OR Balines* OR Sumatra* OR Celebes* OR Sulawesi* OR Madoera* OR Madura*

4. \#1 AND \#2 AND \#3

\section{Data collection}

Two reviewers independently screened the title and the abstract of all retrieved studies. Disagreements between the two reviewers were solved through discussion. Studies without relevant information were excluded. Later, the full texts of the selected articles were assessed for eligibility using the same procedure as the abstract screening.

\section{Quality assessment}

Two reviewers independently assessed the risk of bias by using the Newcastle-Ottawa Scale (NOS) [16]. The final global quality score was discussed by two reviewers.

\section{Data extraction and analysis}

All the included studies were extracted using the modified Cochrane data extraction form. Extracted data included study period, research method, sample size, age group, prevalence, incidence, case fatality rate, 
carrier and carriage rate, etiology, and laboratory method of identification.

\section{Results}

We archived 1,756 publications from our search strategy and included 36 publications for analysis (Figure 1).

\section{Disease Burden}

Five studies and thirteen government publications report on the disease burden related to LRTI in children under five years of age in Indonesia (Table 1) [17-34].
Prevalence

The Ministry of Health of the Republic of Indonesia reports the prevalence of LRTI annually based on healthcare data collected from all provinces in Indonesia [21-24,26-32]. Mean, and standard deviation from 2009 until 2019 reports are $2.26 \%$ and $0.30 \%$, respectively (Figure 2). National Institute of Health Research and Development reported a higher prevalence $(4.5-4.8 \%)$ based on a household survey on 2013 and 2018 [25,34].

Table 1. Summary table of studies reporting disease burden.

\begin{tabular}{|c|c|c|c|c|c|c|c|c|}
\hline Reference & Study period & $\begin{array}{l}\text { Research } \\
\text { method }\end{array}$ & $\begin{array}{l}\text { Study Location (City, } \\
\text { Province, Island) }\end{array}$ & Sample size & Age group & Prevalence & Incidence & $\begin{array}{c}\text { Case Fatality } \\
\text { Rate }\end{array}$ \\
\hline Azmi 2016 & $\begin{array}{l}\text { Data from } \\
2010 \text { and } \\
2011\end{array}$ & $\begin{array}{l}\text { Cross sectional } \\
\text { using Case } \\
\text { mix system }\end{array}$ & NA & $\begin{array}{l}\text { Indonesia: } 42 \\
\text { private and } \\
\text { public hospitals }\end{array}$ & $\begin{array}{l}\text { Children }(0-4 \\
\text { years) and } \\
\text { adults }\end{array}$ & NA & $\begin{array}{l} \pm 5,000 / 100,000 \\
\text { discharge }\end{array}$ & $0-4 \%$ \\
\hline Simoes 2011 & $\begin{array}{l}\text { February } \\
\text { 1999- } \\
\text { Febryary } \\
2001\end{array}$ & Cross sectional & Bandung, West Java, Java & 2,014 & $<5$ years & NA & $\begin{array}{l}38.54-57.25 \text { per } \\
1,000 \text { child-years }\end{array}$ & NA \\
\hline Susilarini 2018 & $\begin{array}{l}\text { May 2013- } \\
\text { April } 2016\end{array}$ & Cross sectional & $\begin{array}{l}\text { Gunung Kidul, Yogyakarta } \\
\text { Special Region, Java; } \\
\text { Balikpapan, East Kalimantan, } \\
\text { Kalimantan; Deli Serdang, } \\
\text { North Sumatera, Sumatera }\end{array}$ & $\begin{array}{l}911 \text { children } \\
\text { from total } 1,527 \\
\text { patients }\end{array}$ & $\begin{array}{l}\text { Children }(0-4 \\
\text { years }) \text {, other } \\
5->70 \text { years }\end{array}$ & NA & $\begin{array}{c}82-114 \text { per } 100,000 \\
\text { population }\end{array}$ & NA \\
\hline Tan 2018 & $\begin{array}{l}\text { January- } \\
\text { December } \\
2011\end{array}$ & Cross sectional & Bandung, West Java, Java & $\begin{array}{l}\text { Indonesia: } 949 \\
\text { episodes }\end{array}$ & $<5$ years & NA & $\begin{array}{c}886 / 949 \text { confirmed } \\
\text { CAP, } 15 / 949 \\
\text { bacterial CAP }\end{array}$ & NA \\
\hline $\begin{array}{l}\text { Ministry of Health } \\
\text { Republic of Indonesia } \\
2009\end{array}$ & 2008 & Cross sectional & NA & $20,887,256$ & $<5$ years & $\begin{array}{c}1.88 \% \\
(392,923 / 20,887,256)\end{array}$ & NA & NA \\
\hline $\begin{array}{l}\text { Ministry of Health } \\
\text { Republic of Indonesia } \\
2010\end{array}$ & 2009 & Cross sectional & NA & $17,402,735$ & $<5$ years & $\begin{array}{c}2.24 \% \\
(390,319 / 17,402,735)\end{array}$ & NA & NA \\
\hline $\begin{array}{l}\text { Ministry of Health } \\
\text { Republic of Indonesia } \\
2011\end{array}$ & 2010 & Cross sectional & NA & $21,704,229$ & $<5$ years & $\begin{array}{c}2.30 \% \\
(499,259 / 21,704,229)\end{array}$ & NA & NA \\
\hline $\begin{array}{l}\text { Ministry of Health } \\
\text { Republic of Indonesia } \\
2012\end{array}$ & 2011 & Cross sectional & NA & $23,315,846$ & $<5$ years & $\begin{array}{c}2.39 \% \\
(559,114 / 23,315,846)\end{array}$ & NA & $\begin{array}{c}0.11 \% \\
(609 / 559,114)\end{array}$ \\
\hline $\begin{array}{l}\text { Ministry of Health } \\
\text { Republic of Indonesia } \\
2013\end{array}$ & 2012 & Cross sectional & NA & $23,471,733$ & $<5$ years & $\begin{array}{c}2.34 \% \\
(549,708 / 23,471,733)\end{array}$ & NA & $\begin{array}{c}0.11 \% \\
(609 / 549,708)\end{array}$ \\
\hline $\begin{array}{l}\text { Ministry of Health } \\
\text { Republic of Indonesia } \\
2014\end{array}$ & 2013 & Cross sectional & NA & $23,366,598$ & $<5$ years & $\begin{array}{c}2.44 \% \\
(571,547 / 23,366,598)\end{array}$ & NA & NA \\
\hline $\begin{array}{l}\text { Ministry of Health } \\
\text { Republic of Indonesia } \\
2015\end{array}$ & 2014 & Cross sectional & NA & $22,311,380$ & $<5$ years & $\begin{array}{c}2.95 \% \\
(657,490 / 22,311,380)\end{array}$ & NA & $\begin{array}{c}0.08 \% \\
(496 / 657,490)\end{array}$ \\
\hline $\begin{array}{l}\text { Ministry of Health } \\
\text { Republic of Indonesia } \\
2016\end{array}$ & 2015 & Cross sectional & NA & $24,625,211$ & $<5$ years & $\begin{array}{c}2.25 \% \\
(554,650 / 24,625,211)\end{array}$ & NA & $\begin{array}{c}0.16 \% \\
(877 / 554,650)\end{array}$ \\
\hline $\begin{array}{l}\text { Ministry of Health } \\
\text { Republic of Indonesia } \\
2017\end{array}$ & 2016 & Cross sectional & NA & $24,520,873$ & $<5$ years & $\begin{array}{c}2.31 \% \\
(568,146 / 24,520,873)\end{array}$ & NA & $\begin{array}{c}0.11 \% \\
(598 / 568,146)\end{array}$ \\
\hline $\begin{array}{l}\text { Ministry of Health } \\
\text { Republic of Indonesia } \\
2018\end{array}$ & 2017 & Cross sectional & NA & $28,142,450$ & $<5$ years & $\begin{array}{c}1.81 \% \\
(511,434 / 28,142,450)\end{array}$ & $\begin{array}{l}20.54 \text { (per } 1,000 \\
\text { children) }\end{array}$ & $\begin{array}{c}0.34 \% \\
(1,752 / 511,434)\end{array}$ \\
\hline $\begin{array}{l}\text { Ministry of Health } \\
\text { Republic of Indonesia } \\
2019\end{array}$ & 2018 & Cross sectional & NA & $25,190,309$ & $<5$ years & $\begin{array}{c}2.00 \% \\
(505,331 / 25,190,309)\end{array}$ & NA & $\begin{array}{c}0.08 \% \\
(425 / 505,331)\end{array}$ \\
\hline $\begin{array}{l}\text { National Institute of } \\
\text { Health Research and } \\
\text { Development MoH } \\
\text { Republic of Indonesia } \\
2013\end{array}$ & $\begin{array}{l}\text { May-June } \\
2013\end{array}$ & Cross sectional & NA & $\begin{array}{l}1,027,763 \\
\text { household }\end{array}$ & $<5$ years & $4.5 \%$ & NA & NA \\
\hline $\begin{array}{l}\text { National Institute of } \\
\text { Health Research and } \\
\text { Development MoH } \\
\text { Republic of Indonesia } \\
2019\end{array}$ & 2018 & Cross sectional & NA & $\begin{array}{c}300,000 \\
\text { household }\end{array}$ & $<5$ years & $4.8 \%$ & NA & NA \\
\hline Purniti 2011 & $\begin{array}{c}\text { December } \\
\text { 2007-January } \\
2009\end{array}$ & Cross sectional & Denpasar, Bali, Bali & 736 patients & $\begin{array}{l}28 \text { days- } 60 \\
\text { months }\end{array}$ & NA & $534.2 / 100,000$ & NA \\
\hline
\end{tabular}

NA: Data not available (not mentioned in the publication). 


\section{Incidence}

Several studies counted the incidence of LRTI. Almost all of them used different denominators: 5,000 per 100,000 discharge, $38.54-57.25$ per 1,000 childyears, $82-114$ per 100,000 population, 534.2 per 100,000 population, 20.54 per 1,000 children [17$19,31,33]$.

\section{Case Fatality Rate (CFR)}

Seven reports from the Ministry of Health of the Republic of Indonesia have CFR data of LRTI (Figure 2) $[24,26,28-32]$. CFR median is $0.11 \%$ (min $0.08 \%$ max $0.34 \%)$. Similar CFR results $(0-4 \%)$ are reported by Azmi et al [17].
Figure 2. Prevalence and case fatality rate (CFR) of LRTI in children under five years of age in Indonesia, data from the Ministry of Health of the Republic of Indonesia.

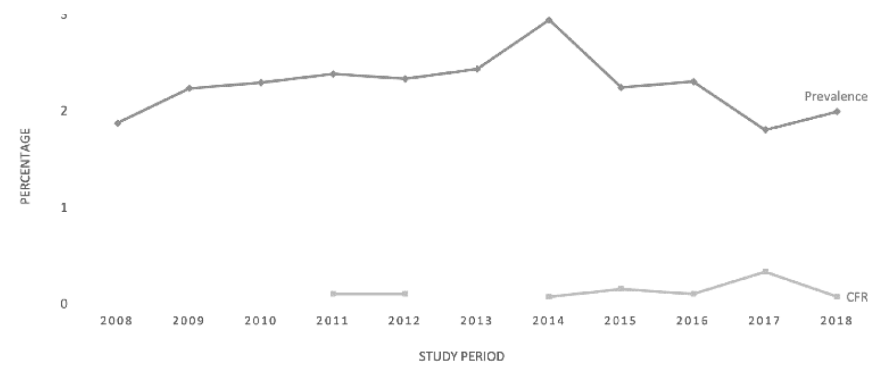

Table 2. Summary table of studies reporting etiology of lower respiratory tract infection.

\begin{tabular}{|c|c|c|c|c|c|c|c|c|}
\hline Reference & Study period & Research method & Setting & $\begin{array}{l}\text { Study Location } \\
\text { (City, Province, } \\
\text { Island) }\end{array}$ & Sample size & Age group & $\begin{array}{c}\text { Etiology } \\
\text { (number of isolates) }\end{array}$ & $\begin{array}{l}\text { Laboratory method } \\
\text { of identification }\end{array}$ \\
\hline Agustiningsih 2012 & July 2008-June 2009 & Cross sectional & $\begin{array}{l}\text { Hospital (not } \\
\text { specified) }\end{array}$ & $\begin{array}{c}\text { Jakarta, Special } \\
\text { Capital Region, Java }\end{array}$ & $\begin{array}{l}62 \text { children }<5 \text { years } \\
\text { from } 230 \text { patients }\end{array}$ & $\begin{array}{c}\text { Children }(<15 \text { years } \\
\text { of age) and adults } \\
(>15 \text { years old })\end{array}$ & $\begin{array}{l}\text { K. pneumoniae }(15), \\
\text { S. pneumoniae }(9), \\
\text { Influenza A (3), } \\
\text { mixed virus-bacteria } \\
(21), \text { mixed bacteria- } \\
\text { bacteria (16) }\end{array}$ & $\begin{array}{l}\text { Multiplex PCR } \\
\text { (Seeplex } \Re), \text { real } \\
\text { time PCR }\end{array}$ \\
\hline Indawati 2014 & $\begin{array}{c}\text { January- October } \\
2010\end{array}$ & Cross sectional & $\begin{array}{l}\text { Hospital (not } \\
\text { specified) and } \\
\text { primary health } \\
\text { center }\end{array}$ & $\begin{array}{c}\text { Jakarta, Special } \\
\text { Capital Region, Java }\end{array}$ & $\begin{array}{l}105 \text { children } 0-5 \\
\text { years from total } 167 \\
\text { patients }\end{array}$ & $\begin{array}{c}0-5 \text { years and } 6-14 \\
\text { years }\end{array}$ & $\begin{array}{l}\text { Influenza A (2), } \\
\text { Influenza B (12) }\end{array}$ & $\begin{array}{c}\text { Rapid test } \\
\text { (BinaxNOW'T } \\
\text { Influenza A and B } \\
\text { Test) }\end{array}$ \\
\hline Nataprawira 2015 & $\begin{array}{c}\text { Data from October } \\
\text { 2008-December } \\
2014\end{array}$ & Cross sectional & $\begin{array}{l}\text { Hospital (not } \\
\text { specified) }\end{array}$ & $\begin{array}{l}\text { Bandung, West Java, } \\
\text { Java }\end{array}$ & 63 patients & $0-50$ months & B. pertussis (2) & culture \\
\hline Prawira 2012 & $\begin{array}{l}\text { Medical record form } \\
\text { September 2005- } \\
\text { August } 2010\end{array}$ & Cross sectional & $\begin{array}{l}\text { Hospital (not } \\
\text { specified) }\end{array}$ & $\begin{array}{c}\text { Jakarta, Special } \\
\text { Capital Region, Java }\end{array}$ & $\begin{array}{c}3 \text { children }<5 \text { years } \\
\text { from total } 26 \\
\text { participants }\end{array}$ & $\begin{array}{l}\text { Children }(0-18 \\
\text { years) and adults } \\
(>18 \text { years })\end{array}$ & H5N1 (3) & PCR \\
\hline Sari 2017 & $\begin{array}{l}\text { Laboratory record } \\
\text { January 2014- } \\
\text { December } 2015\end{array}$ & Cross sectional & Hospital (NICU) & $\begin{array}{c}\text { NA, Central Java, } \\
\text { Java }\end{array}$ & 225 patients & neonates & K. pneumoniae (49) & NA \\
\hline Simoes 2011 & $\begin{array}{l}\text { February } 1999- \\
\text { Febryary } 2001\end{array}$ & Cross sectional & $\begin{array}{c}\text { Primary health } \\
\text { center }\end{array}$ & $\begin{array}{l}\text { Bandung, West Java, } \\
\text { Java }\end{array}$ & 2,014 & $<5$ years & RSV (163) & $\begin{array}{l}\text { Abbott Test Pak, } \\
\text { real time reverse } \\
\text { transcription PCR }\end{array}$ \\
\hline Susilarini 2018 & $\begin{array}{l}\text { May 2013-April } \\
2016\end{array}$ & Cross sectional & $\begin{array}{l}\text { Hospital (not } \\
\text { specified) }\end{array}$ & $\begin{array}{c}\text { Gunung Kidul, } \\
\text { Yogyakarta Special } \\
\text { Region, Java; } \\
\text { Balikpapan, East } \\
\text { Kalimantan, } \\
\text { Kalimantan; Deli } \\
\text { Serdang, North }\end{array}$ & $\begin{array}{l}911 \text { children from } \\
\text { total } 1,527 \text { patients }\end{array}$ & $\begin{array}{c}\text { Children }(0-4 \\
\text { years), other 5->70 } \\
\text { years }\end{array}$ & Influenza virus (114) & $\begin{array}{l}\text { Real time reverse } \\
\text { transcription PCR }\end{array}$ \\
\hline Yuliarti 2012 & $\begin{array}{c}\text { June 2008-December } \\
2009\end{array}$ & Cross sectional & $\begin{array}{l}\text { Hospital (not } \\
\text { specified) }\end{array}$ & $\begin{array}{l}\text { Sumatera, Sumatera } \\
\text { Jakarta, Special } \\
\text { Capital Region, Java }\end{array}$ & 205 patients & $\begin{array}{l}28 \text { days to } 60 \\
\text { months }\end{array}$ & S. pneumoniae (1) & $\begin{array}{c}\text { Conventional } \\
\text { biochemical test }\end{array}$ \\
\hline Widoretno 2012 & $\begin{array}{l}\text { March- December } \\
2008\end{array}$ & Cross sectional & $\begin{array}{l}\text { Hospital (not } \\
\text { specified) }\end{array}$ & $\begin{array}{c}\text { Jakarta, Special } \\
\text { Capital Region, } \\
\text { Java; Bandung, West } \\
\text { Java, Java; } \\
\text { Semarang, Central } \\
\text { Java, Java; } \\
\text { Makassar, South } \\
\text { Sulawesi, Sulawesi; } \\
\text { Kupang, East Nusa } \\
\text { Tenggara, Timor; } \\
\text { Tangerang, Banten, } \\
\text { Java }\end{array}$ & $\begin{array}{l}215 \text { throat specimens } \\
\text { from } 350 \text { patients }\end{array}$ & $<5$ years & $\begin{array}{c}\text { Influenza A (4), } \\
\text { RSV (20), } \\
\text { Adenovirus (1), } \\
\text { Bocavirus (2), } \\
\text { Coronavirus (2), } \\
\text { Metapneumo virus } \\
\text { (2), Parainfluenza } \\
\text { virus (6), Rhinovirus } \\
\text { (10), Coxsackie- } \\
\text { Entero virus (19), } \\
\text { Entero-Rhino virus } \\
\text { (10), Coxsackie- } \\
\text { Entero-Rhino virus } \\
\text { (3), Entero-Adeno } \\
\text { virus (1), Entero } \\
\text { virus-RSV (4), RSV- } \\
\text { Corona virus (1), } \\
\text { RSV-Enterovirus- } \\
\text { Rhinovirus (4) }\end{array}$ & $\begin{array}{c}\text { Multiplex Bead } \\
\text { Array Assay } \\
\text { ResPlex II v.2, } \\
\text { Luminex } \mathbb{} \text {, }\end{array}$ \\
\hline Setyati 2012 & $\begin{array}{l}\text { Medical records } \\
\text { January-June } 2011\end{array}$ & Cross sectional & Hospital (PICU) & $\begin{array}{c}\text { Yogyakarta, } \\
\text { Yogyakarta Special } \\
\text { Region, Java }\end{array}$ & 95 bacterial isolates & $\begin{array}{c}\text { Children }(<1 \text { and } \geq 1 \\
\text { year })\end{array}$ & $\begin{array}{c}\text { Klebsiella } \\
\text { pneumoniae }(15)\end{array}$ & NA \\
\hline Gatera 2014 & $\begin{array}{l}\text { Medical record } \\
\text { January 2011- } \\
\text { December } 2012\end{array}$ & Cross sectional & $\begin{array}{l}\text { Hospital (not } \\
\text { specified) }\end{array}$ & $\begin{array}{c}\text { Bandung, West Java, } \\
\text { Java }\end{array}$ & 24 bacterial isolates & $1-5$ years & $\begin{array}{c}\text { Klebsiella } \\
\text { pneumoniae }(7), \\
\text { Streptococcus } \\
\text { pneumoniae }(9)\end{array}$ & NA \\
\hline Purniti 2011 & $\begin{array}{l}\text { December 2007- } \\
\text { January } 2009\end{array}$ & Cross sectional & $\begin{array}{l}\text { Hospital (not } \\
\text { specified) }\end{array}$ & Denpasar, Bali, Bali & 736 patients & 28 days- 60 months & $\begin{array}{c}\text { Klebsiella } \\
\text { pneumoniae }(1)\end{array}$ & NA \\
\hline
\end{tabular}

NA: Data not available (not mentioned in the publication); NICU: Neonatal Intensive Care Unit; PICU: Pediatric Intensive Care Unit. 


\section{Etiology}

Twelve studies described the etiology of LRTI in children under five years of age in Indonesia (Table 2) $[18,19,33,35-43]$. The studies were conducted in different settings (hospital based and/ or primary health care based). In addition, different laboratory method of identification was used. Five studies focused on viral pathogens, while six studies focused on bacteria as the main pathogen $[18,19,36,38,41]$. Only one study described both bacterial and viral [35]. Figure 3 shows the location and indicates the sample size of the different studies reporting on etiology of lower respiratory tract infection in children under five years of age in Indonesia.

$\underline{\text { Viral }}$

Based on the number of studies and the number of cases described in the separate studies, influenza virus was the most common cause of viral respiratory tract infections in children under five years of age in Indonesia. Other viruses prevalent in this age group and mentioned in the different studies were respiratory syncytial virus, coxsackievirus, enterovirus, coronavirus, adenovirus, parainfluenza virus, bocavirus, rhinovirus, and human metapneumovirus $[35,41]$.

\section{Influenza Virus}

Data regarding influenza virus infections in Indonesian children were found in five different studies
$[19,35,36,38,41]$. Influenza A virus was the most commonly reported type. The studies identified not only H1 and H3 subtypes but also H5 [19,35,38,41]. One study conducted in an infectious disease hospital in Jakarta analyzed the clinical, laboratory, and radiologic characteristics of confirmed avian influenza (H5N1) cases. There were three patients under five years of age from all thirteen children. Two out of three died. The main clinical conditions were fever, productive cough, and dyspnea. The cause of death was acute respiratory distress syndrome [38]. Another study, also conducted in Jakarta, found influenza B in twelve patients among 167 children age $0-14$ years diagnosed with influenzalike-illness and pneumonia [36].

\section{$R S V$}

Two studies identified RSV as the causative agent of LRTI [18,41]. One large study in Bandung, West Java, consist of 2,014 participants under five years of age, revealed that in Indonesia, the incidence of LRTI due to RSV was relatively low. No RSV found as the etiology of LRTI in children under two months of age [18].

\section{$\underline{\text { Bacterial }}$}

All studies that reported bacteria as the cause of LRTI in children under five years of age in Indonesia were hospital-based. All studies were conducted in Java Island, except two studies located in Bali and Sumatera $[33,43]$.

Figure 3. Location and representation of total sample size of studies reporting etiology of lower respiratory tract infection.

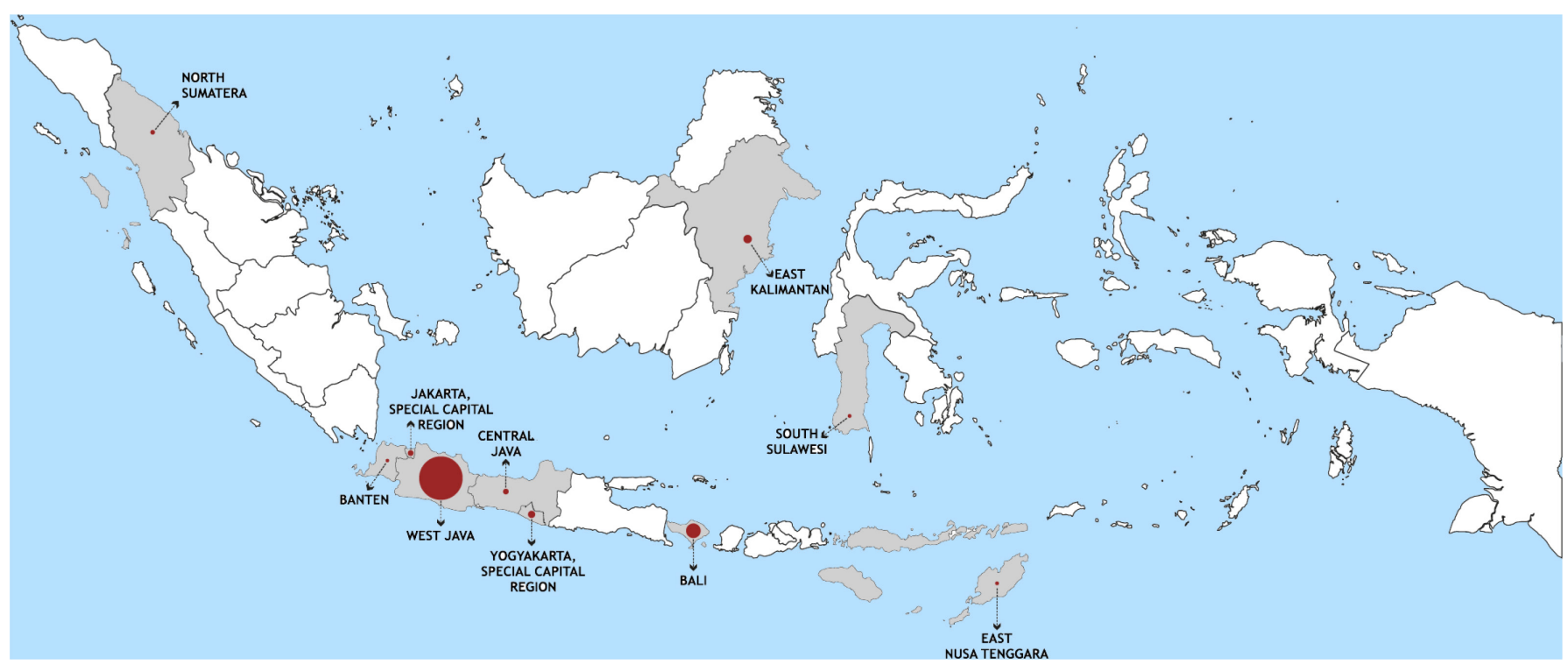

West Java 2171, Bali 736, East Kalimantan 417, Jakarta 396, Yogyakarta 356, Central Java 276, North Sumatera 221, South Sulawesi 42, East Nusa Tenggara 13, Banten 8 . 


\section{K. pneumoniae}

$K$. pneumoniae was the most commonly reported bacterial pathogens $[33,35,39,42,43] \mathrm{K}$. pneumoniae isolate was found in the blood of children with respiratory complaints $[33,42,43]$, the tracheal aspirate $[35,42]$, nasal-nasopharyngeal swab, and bronchoalveolar lavage [35].

\section{S. pneumoniae}

Only three out of twelve studies identified $S$. pneumoniae, 19 cases in total $[35,40,43]$. Two studies identified it from a blood culture sample (see Invasive Disease). The third study used multiplex PCR to detect $S$. pneumoniae among hospitalized-suspected H5N1 patients. In this study, the prevalence of $S$. pneumoniae co-occurring or co-infecting with $H$. influenzae and $K$. pneumoniae was higher than a single infection with $S$. pneumoniae [35].

\section{B. pertussis}

One study retrospectively identified $B$. pertussis associated with pertussis-like symptoms in sixty-one probable and two confirmed cases. All patients were in the paroxysmal phase when they came to the hospital; thus, only a limited number of $B$. pertussis could be detected. Seventy-nine percent of the case was at the age of 0-5 months. Only two patients had a history of pertussis vaccination. No fatality found. Unfortunately, household contacts could not be screened, and no other information related to a possible outbreak was reported [37].

Viral-bacterial co-infection

One study conducted in Jakarta aimed to find the etiology of lower respiratory tract infection in 108 children and 122 adults. The authors reported polymicrobial infections, both viral and bacterial pathogens, as measured in the nasal swab, throat swab, tracheal aspirates, and bronchoalveolar lavage. From this study, it was concluded that bacterial-viral coinfection, in particular K. pneumoniae and Influenza A, was the most common cause, followed by bacterial coinfection (S. pneumoniae and H. influenzae) [35].

\section{Bacterial carriage}

Studies focused on the bacterial carriage in the upper respiratory tract of children under five years of age were done in Java, Lombok, and Sumatera island (Table 3) [8-12,44-47]. Figure 4 shows the location and indicates the sample size of the different studies reporting on bacterial carriage in children under five years of age in Indonesia.

\section{S. pneumoniae}

Eight studies reported S. pneumoniae carriage [8$12,44,45,47]$. The lowest rate in healthy children from two to twelve months old was $22 \%$. The same study showed that the prevalence could increase to $68.4 \%$ in

Table 3. Summary table of studies reporting bacterial carriage.

\begin{tabular}{|c|c|c|c|c|c|c|c|}
\hline Reference & Study period & Research method & $\begin{array}{c}\text { Study Location } \\
\text { (Region, Province, } \\
\text { Island) } \\
\end{array}$ & Sample size & Age group & Carrier, carriage rate & $\begin{array}{l}\text { Laboratory method of } \\
\text { identification }\end{array}$ \\
\hline Dunne 2018 & February- March 2016 & Cross sectional & $\begin{array}{l}\text { Bandung, West Java, } \\
\text { Java; Central Lombok, } \\
\text { West Nusa Tenggara, } \\
\text { Lombok; Padang, West } \\
\text { Sumatera, Sumatera }\end{array}$ & 302 healthy children & 12-24 months & $\begin{array}{l}\text { S. pneumoniae }(49.5 \%) \text {, } \\
\text { H. influenzae }(27.5 \%) \text {, } \\
\text { M. catarrhalis }(42.7 \%) \text {, } \\
\text { S. aureus }(7.3 \%)\end{array}$ & qPCR \\
\hline Fadlyana 2018 & NA & Cross sectional & $\begin{array}{l}\text { Bandung, West Java, } \\
\text { Java; Lombok, West } \\
\text { Nusa Tenggara, } \\
\text { Lombok; Padang, West } \\
\text { Sumatera, Sumatera }\end{array}$ & 302 healthy children & $12-24$ months & $\begin{array}{c}\text { S. pneumoniae } \\
\text { (Padang: } 34.6 \% \text {, } \\
\text { Bandung: } 64.0 \% \text {, } \\
\text { Lombok: } 50.5 \% \text { ), } M \text {. } \\
\text { catarrhalis (Padang: } \\
31.7 \% \text {, } \\
\text { Bandung: } 45.0 \% \text {, } \\
\text { Lombok } 51.5 \% \text {, }\end{array}$ & $\begin{array}{l}\text { qPCR; FTD Bacterial } \\
\text { pneumoniae CAP } \\
\text { qPCR kit, Fast Tract } \\
\text { Diagnostic }\end{array}$ \\
\hline Farida 2013 & February- April 2010 & Cross sectional & $\begin{array}{l}\text { Semarang, Central } \\
\text { Java, Java }\end{array}$ & $\begin{array}{l}243 \text { healthy children, } \\
253 \text { healthy adults }\end{array}$ & $\begin{array}{c}\text { Children (6-60 months) } \\
\text { and adults }\end{array}$ & $\begin{array}{c}\text { K. pneumoniae }(7 \%) \text {, } \\
\text { S. pneumoniae }(43 \%) \text {, } \\
\text { other Gram-Negative } \\
\text { Bacteria }(20 \%)\end{array}$ & $\begin{array}{c}\text { VITEK® 2, optochin } \\
\text { test }\end{array}$ \\
\hline Farida 2014 & February- April 2010 & Cross sectional & $\begin{array}{c}\text { Semarang, Central } \\
\text { Java, Java } \\
\text { Central Lombok, West }\end{array}$ & $\begin{array}{l}243 \text { healthy children, } \\
253 \text { healthy adults }\end{array}$ & $\begin{array}{c}\text { Children (6-60 months) } \\
\text { and adults }\end{array}$ & S. pneumoniae $(43 \%)$ & $\begin{array}{l}\text { Optochin test, DNA } \\
\text { hybridization }\end{array}$ \\
\hline Hadinegoro 2016 & 2012 & Cross sectional & $\begin{array}{c}\text { Nusa Tenggara, } \\
\text { Lombok }\end{array}$ & 1,200 healthy children & 2 to 60 months & S. pneumoniae $(46 \%)$ & Optochin test \\
\hline Murad 2019 & $\begin{array}{l}\text { November 2014- } \\
\text { January } 2015\end{array}$ & Cohort & $\begin{array}{c}\text { Bandung, West Java, } \\
\text { Java }\end{array}$ & 200 healthy infants & $\begin{array}{l}2 \text { months (follow up } \\
\text { until } 12 \text { months) }\end{array}$ & $\begin{array}{l}\text { S. pneumoniae ( } 22 \% \text { at } \\
2 \text { months and } 68.4 \% \text { at } \\
12 \text { months of age) }\end{array}$ & qPCR \\
\hline Safari 2014 & January- July 2012 & Cross sectional & $\begin{array}{c}\text { Jakarta, Special Capital } \\
\text { Region, Java }\end{array}$ & 90 HIV patients & 4-144 months & S. pneumoniae $(46 \%)$ & PCR \\
\hline Hatmaningtyas 2013 & May- June 2013 & Cross sectional & $\begin{array}{l}\text { Semarang, Central } \\
\text { Java, Java }\end{array}$ & 174 & 6-60 months & $\begin{array}{c}\text { K. pneumoniae }(0- \\
2.9 \%)\end{array}$ & Biochemical test \\
\hline Hikmawati 2010 & February- March 2010 & Cross sectional & $\begin{array}{l}\text { Semarang, Central } \\
\text { Java, Java }\end{array}$ & $\begin{array}{l}75 \text { children from total } \\
150 \text { participants }\end{array}$ & $\begin{array}{l}\text { Children ( } 0-5 \text { years) } \\
\text { and adults ( } 45-70 \text { year) }\end{array}$ & $\begin{array}{c}\text { S. pneumoniae }(45.3 \%), \\
\text { H. influenzae }(12 \%), \\
\text { Enterobacteriaceae } \\
(10.7 \%), \text { S. aureus } \\
(6.7 \%), \text { M. catarrhalis } \\
(5.3 \%)\end{array}$ & Biochemical test \\
\hline
\end{tabular}


children of 12 months or older [45]. Serotype data was found in five studies [9-12,45]. The PCV 13 serotype carriage rate was 9.1 to $35.1 \%$ [45]. Three other studies in children from two to sixty months of age reported a PCV 13 coverage of $45 \%, 56 \%$, and $60 \%$ [10-12]. Three of the five studies found $6 \mathrm{~A} / \mathrm{B}$ serotype as the most prevalent serotype in Indonesian children $[10,12,45]$. Other serotypes that were frequently found in all five studies were vaccine types $19 \mathrm{~F}$ and $23 \mathrm{~F}$ and non-vaccine types 2 and 15B/C [9-12,45].

\section{H. influenzae}

H. influenzae carriage rates ranged from $12 \%$ up to $32.9 \%$ in two studies $[9,47]$. One of these studies serotyped $H$. influenzae isolate, and none of the 83 isolates was $H$. influenzae type $\mathrm{B}$, which reflects the effect of the Hib vaccine on a carriage [9].

\section{M. catarrhalis}

One study in Semarang, Central Java, reports the carriage rate of $M$. catarrhalis from children under five years of age at $5.3 \%$. The prevalence of $M$. catarrhalis from healthy children aged 12-24 months in Lombok, West Nusa Tenggara, was ten times higher than Semarang, approximately, making it the highest also compared to Bandung and Padang, West Sumatra $[9,44,47]$.

\section{S. aureus}

$S$. aureus prevalence was the lowest compared to $S$. pneumoniae, $H$. influenzae, and $M$. catarrhalis. The prevalence was between $6.7 \%$ until approximately
9.0\%. S. aureus had the same prevalence in Bandung as in Central Lombok (around 8.0\%) [9,47].

Other bacterial pathogens identified in carriage studies

Semarang was the only place where $K$. pneumoniae, Pseudomonas spp. and A. baumannii comp. were identified in asymptomatic carriers. The range of carriage rates of $K$. pneumoniae varied from $2.9 \%$ to $7.0 \%$ [8,46]. Pseudomonas spp. and A. baumannii comp. were found in $7 \%$ and $5 \%$ of the carriers, respectively [8].

\section{Invasive Disease}

Three studies located in Denpasar, Bandung, and Jakarta showed the burden of invasive pneumococcal disease (IPD) in Indonesia [33,40,43]. Two studies directly aimed to measure the burden of invasive pneumococcal disease $[33,40]$. No pneumococci were detected in the 736 IPD and pneumonia patients in Denpasar [33]. A study in Bandung found nine pneumococcal isolates in blood cultures from 486 patients with pneumonia [43]. A study in Jakarta found one IPD case from 205 patients diagnosed with pneumonia, meningitis, sepsis, and suspected occult bacteremia. The IPD patient was a three-month-old baby boy infected with $S$. pneumoniae serotype 7F, had bilateral lobar pneumonia and meningitis and died on the fifth day of hospitalization because of respiratory failure [40].

Figure 4. Location and representation of total sample size of studies reporting bacterial carriage.

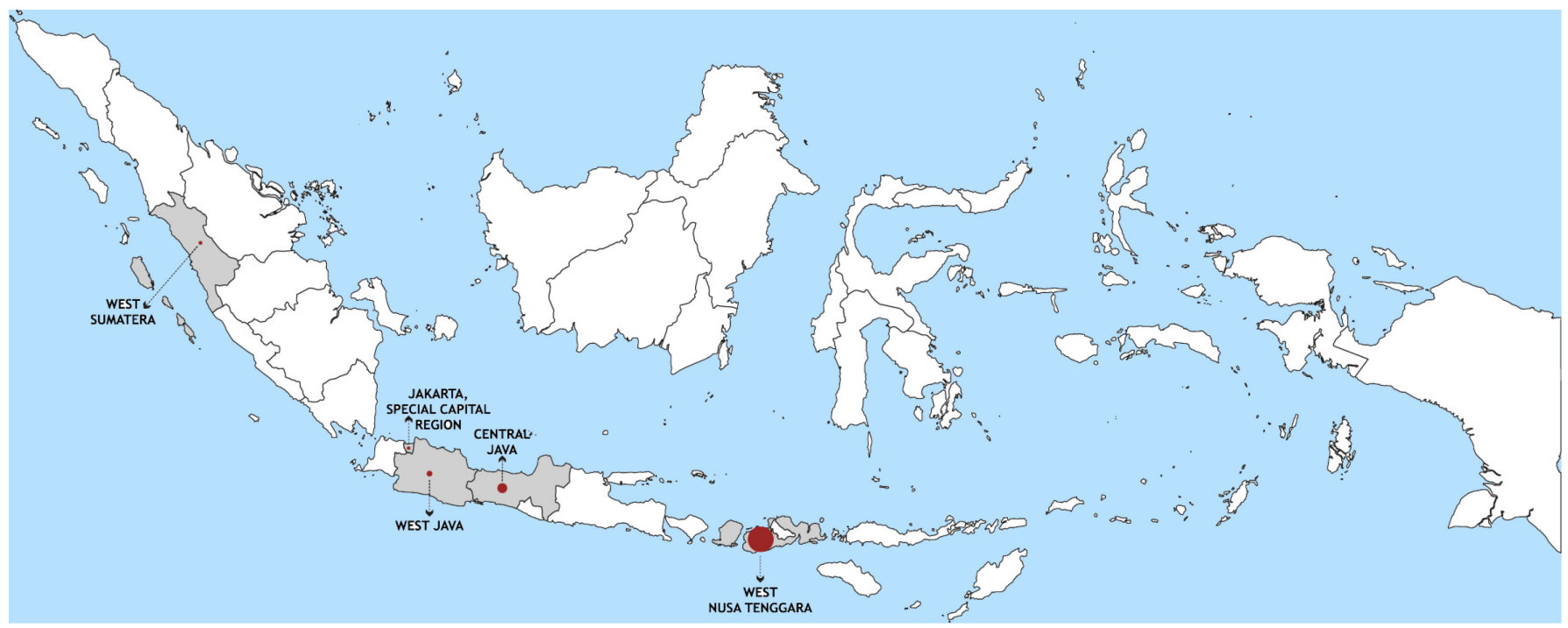

West Nusa Tenggara 1301, Central Java 492, West Java 300, West Sumatera 101, Jakarta 90. 


\section{Discussion}

The objective of this study was to collect all available data to have a complete overview of the current status of the burden and etiology of LRTI in children under five years of age in Indonesia. Furthermore, we obtained all available information about asymptomatic carriage of well-known LRTI pathogens and about invasive infections associated with respiratory complaints in this population, which are strongly related to LRTI and therefore important to include in this review.

The median CFR of LRTI in children under five years of age in Indonesia from 2011-2018 was 0.11\% as reported by the Ministry of Health of the Republic of Indonesia [21-24,26-32]. This is relatively low as compared to other countries such as Malawi (6.6\%) and India $(8.2 \%)[48,49]$.

We found that the influenza virus was the most commonly reported viral cause of LRTI in children under five years of age in Indonesia. Although this result differs from other studies performed in other populations, which found that RSV is most common in children under five years of age [50-52]. K. pneumoniae was the most commonly reported bacterial cause of LRTI. This has earlier been found in other populations, as reported in a study conducted in China [53].

In healthy asymptomatic Indonesian children under five years of age, not vaccinated with a pneumococcal vaccine, $S$. pneumoniae had the highest prevalence rate. The highest carrier rate reported was $68.4 \%$ [45]. These data are in line with a previous study in Thailand, showing a carriage rate of $67.6-83.6 \%$ [54]. Although we noticed substantial carriage rates with $S$. pneumoniae in healthy children under five years of age in Indonesia, it is not the most commonly identified bacterial cause of LRTI in children under five years of age in Indonesia. This is likely an underrepresentation due to the use of insensitive diagnostic methods. Interestingly $K$. pneumoniae, the most commonly reported cause of LRTI, is also found in asymptomatic carriers in Indonesia. It is speculated that this is due to the consumption of contaminated food and water [8]. The coverage of vaccine serotypes (based on PCV13) was between $45-60 \%$. A similar conclusion was reached from a study reporting prevalence of $S$. pneumoniae in South East Asia, in which a coverage of $65 \%$ was found [55].

Unfortunately, there is a lack of information regarding the incidence of LRTI-related invasive disease in children under five years of age in Indonesia. Therefore no conclusions can be drawn based on the available literature. This strongly demands for more studies identifying the etiology of LRTI-related invasive disease.

Although we only included a total of 36 English and Indonesian articles, we are confident that this is all relevant literature available at this moment. Reviewing this literature gives a better understanding of what is known about the burden and etiology of LRTI in children in Indonesia. However, at the same time we have to conclude that there is an important lack of information regarding the burden and etiology of LRTI, especially because most studies are done in certain regions of Java, while there is hardly any information available from other regions. Based on this review we recommend to improve the diagnostics of respiratory tract infections in children in Indonesia and to enhance the capacity of infectious disease surveillance, in particular in other provinces than Jakarta, West and Central Java.

\section{Conclusions}

Surveillance and diagnostic studies are urgently needed and should be conducted in different parts of Indonesia to improve insight in the burden and etiology of LRTI in Indonesia. These data are pivotal to increase the effectiveness of public health strategies, including vaccination and prevention of antimicrobial resistance.

\section{Acknowledgements}

We address our appreciation to Alice H.J. Tillema who build a search strategy for this study.

\section{References}

1. Liu L, Oza S, Hogan D, Chu Y, Perin J, Zhu J, Lawn JE, Cousens S, Mathers C, Black RE. (2016) Global, regional, and national causes of under-5 mortality in 2000-15: an updated systematic analysis with implications for the Sustainable Development Goals. Lancet 388: 3027-3035.

2. McAllister DA, Liu L, Shi T, Chu Y, Reed C, Burrows J, Adeloye D, Rudan I, Black RE, Campbell H, Nair H (2019) Global, regional, and national estimates of pneumonia morbidity and mortality in children younger than 5 years between 2000 and 2015: a systematic analysis. Lancet Glob Health 7: e47-e57.

3. Gessner BD, Sutanto A, Linehan M, Djelantik IGG, Fletcher T, Gerudug IK, Ingerani, Mercer D, Moniaga V, Moulton LH, Mulholland K, Nelson C, Soemohardjo S, Steinhoff M, Widjaya A, Stoeckel P, Maynard J, Arjoso S (2005) Incidences of vaccine-preventable Haemophilus influenzae type $b$ pneumonia and meningitis in Indonesian children: hamletrandomised vaccine-probe trial. Lancet, 365: 43-52.

4. Broughton EI (2007) Economic evaluation of Haemophilus influenzae type $B$ vaccination in Indonesia: a costeffectiveness analysis. J Public Health (Oxf) 29: 441-448.

5. Gessner BD, Sedyaningsih ER, Griffiths UK, Sutanto A, Linehan M, Mercer D, Mulholland EK, Walker DG, Steinhoff 
M, Nadjib M (2008) Vaccine-preventable haemophilus influenza type $\mathrm{B}$ disease burden and cost-effectiveness of infant vaccination in Indonesia. Pediatr Infect Dis J 27: 438443.

6. Nelson CM, Sutanto A, Gessner BD, Suradana IG, Steinhoff MC, Arjoso S (2000) Age- and cause-specific childhood mortality in Lombok, Indonesia, as a factor for determining the appropriateness of introducing Haemophilus influenzae type $b$ and pneumococcal vaccines. J Health Popul Nutr 18: 131-138.

7. Gessner BD, Sutanto A, Steinhoff M, Soewignjo S, Widjaya A, Nelson C, Arjoso S (1998) A population-based survey of Haemophilus influenzae type $b$ nasopharyngeal carriage prevalence in Lombok Island, Indonesia. Pediatr Infect Dis J 17: S179-182.

8. Farida H, Severin JA, Gasem MH, Keuter M, van den Broek P, Hermans PW, Wahyono H, Verbrugh HA (2013) Nasopharyngeal carriage of Klebsiella pneumoniae and other Gram-negative bacilli in pneumonia-prone age groups in Semarang, Indonesia. J Clin Microbiol 51: 1614-1616.

9. Dunne EM, Murad C, Sudigdoadi S, Fadlyana E, Tarigan R, Indriyani SAK, Pell CL, Watts E, Satzke C, Hinds J, Dewi NE, Yani FF, Rusmil K, Mulholland EK, Kartasasmita C (2018) Carriage of Streptococcus pneumoniae, Haemophilus influenzae, Moraxella catarrhalis, and Staphylococcus aureus in Indonesian children: A cross-sectional study. PLoS One 13: e0195098. doi:10.1371/journal.pone.0195098

10. Farida H, Severin JA, Gasem MH, Keuter M, Wahyono H, van den Broek P, Hermans PW, Verbrugh HA (2014) Nasopharyngeal carriage of Streptococcus pneumonia in pneumonia-prone age groups in Semarang, Java Island, Indonesia. PLoS One 9: e87431.

11. Safari D, Kurniati N, Waslia L, Khoeri MM, Putri T, Bogaert D, Trzcinski K (2014) Serotype distribution and antibiotic susceptibility of Streptococcus pneumoniae strains carried by children infected with human immunodeficiency virus. PLoS One 9: e110526.

12. Hadinegoro SR, Prayitno A, Khoeri MM, Djelantik IG, Dewi NE, Indriyani SA, Muttaqin Z, Mudaliana S, Safari D (2016) Nasopharyngeal Carriage of Streptococcus Pneumoniae in Healthy Children under Five Years Old in Central Lombok Regency, Indonesia. Southeast Asian J Trop Med Public Health 47: 485-493.

13. Soewignjo S, Gessner BD, Sutanto A, Steinhoff M, Prijanto M, Nelson C, Widjaya A, Arjoso S (2001) Streptococcus pneumoniae nasopharyngeal carriage prevalence, serotype distribution, and resistance patterns among children on Lombok Island, Indonesia. Clin Infect Dis 32 1039-1043.

14. National Institute of Health Research and Development Ministry of Health Republic of Indonesia (2015) Action plan for health research and development program 2015-2019. Available:https://e-renggar.kemkes.go.id/file2018/eperformance/1-653572-4tahunan.pdf. Accessed 17 October 2019. [Report in Indonesian].

15. Moher D, Liberati A, Tetzlaff J, Altman DG, Group P (2009) Preferred reporting items for systematic reviews and metaanalyses: the PRISMA statement. PLoS Med 6: e1000097.

16. Wells G, Shea B, O'Connell D, Peterson J, Welch V, Losos M, Tugwell P (2012) The Newcastle-Ottawa Scale (NOS) for assessing the quality of nonrandomised studies in metaanalyses.

Available:http://www.ohri.ca/programs/clinical_epidemiolog y/oxford.asp. Accessed: 02 October 2019.
17. Azmi S, Aljunid SM, Maimaiti N, Ali AA, Muhammad Nur A, De Rosas-Valera M, Encluna J, Mohamed R, Wibowo B, Komaryani K, Roberts C (2016) Assessing the burden of pneumonia using administrative data from Malaysia, Indonesia, and the Philippines. Int J Infect Dis 49: 87-93.

18. Simoes EA, Mutyara K, Soh S, Agustian D, Hibberd ML, Kartasasmita CB (2011) The epidemiology of respiratory syncytial virus lower respiratory tract infections in children less than 5 years of age in Indonesia. Pediatr Infect Dis J 30: 778-784.

19. Susilarini NK, Haryanto E, Praptiningsih CY, Mangiri A, Kipuw N, Tarya I, Rusli R, Sumardi G, Widuri E, Sembiring MM, Noviyanti W, Widaningrum C, Lafond KE, Samaan G, Setiawaty V (2018) Estimated incidence of influenzaassociated severe acute respiratory infections in Indonesia, 2013-2016. Influenza Other Respir Viruses 12: 81-87.

20. Tan KK, Dang DA, Kim KH, Kartasasmita C, Kim HM, Zhang XH, Shafi F, Yu TW, Ledesma E, Meyer N (2018) Burden of hospitalized childhood community-acquired pneumonia: A retrospective cross-sectional study in Vietnam, Malaysia, Indonesia and the Republic of Korea. Hum Vaccin Immunother 14: 95-105.

21. Ministry of Health Republic of Indonesia (2009) Indonesia health profile $2008 . \quad$ Available: https://pusdatin.kemkes.go.id/resources/download/pusdatin/pr ofil-kesehatan-indonesia/profil-kesehatan-indonesia-2008.pdf. Accessed 11 October 2019. [Report in Indonesian].

22. Ministry of Health Republic of Indonesia (2010) Indonesia health profile $2009 . \quad$ Available: https://pusdatin.kemkes.go.id/resources/download/pusdatin/pr ofil-kesehatan-indonesia/profil-kesehatan-indonesia-2009.pdf. Accessed 11 October 2019. [Report in Indonesian].

23. Ministry of Health Republic of Indonesia (2011) Indonesia health profile $2010 . \quad$ Available: https://pusdatin.kemkes.go.id/resources/download/pusdatin/pr ofil-kesehatan-indonesia/profil-kesehatan-indonesia-2010.pdf. Accessed 11 October 2019. [Report in Indonesian].

24. Ministry of Health Republic of Indonesia (2012) Indonesia health profile 2011. Available: https://pusdatin.kemkes.go.id/resources/download/pusdatin/pr ofil-kesehatan-indonesia/profil-kesehatan-indonesia-2011.pdf. Accessed 11 October 2019. [Report in Indonesian].

25. National Institute of Health Research and Development Ministry of Health Republic of Indonesia (2013) Basic health research 2013. Available: http://labdata.litbang.kemkes.go.id/images/download/laporan/ RKD/2013/Laporan_riskesdas_2013_final.pdf. Accessed 11 October 2019. [Report in Indonesian].

26. Ministry of Health Republic of Indonesia (2013) Indonesia health profile $2012 . \quad$ Available: https://pusdatin.kemkes.go.id/resources/download/pusdatin/pr ofil-kesehatan-indonesia/profil-kesehatan-indonesia-2012.pdf. Accessed 11 October 2019. [Report in Indonesian].

27. Ministry of Health Republic of Indonesia (2014) Indonesia health profile 2013. Available: https://pusdatin.kemkes.go.id/resources/download/pusdatin/pr ofil-kesehatan-indonesia/profil-kesehatan-indonesia-2013.pdf. Accessed 11 October 2019. [Report in Indonesian].

28. Ministry of Health Republic of Indonesia (2015) Indonesia health profile 2014. Available:https://pusdatin.kemkes.go.id/resources/download/p usdatin/profil-kesehatan-indonesia/profil-kesehatan- 
indonesia-2014.pdf. Accessed 11 October 2019. [Report in Indonesian].

29. Ministry of Health Republic of Indonesia (2016) Indonesia health profile 2015.2 Available: https://pusdatin.kemkes.go.id/resources/download/pusdatin/pr ofil-kesehatan-indonesia/Profil-Kesehatan-Indonesia-Tahun2015.pdf. Accessed 11 October 2019. [Report in Indonesian].

30. Ministry of Health Republic of Indonesia (2017) Indonesia health profile $2016 . \quad$ Available: https://pusdatin.kemkes.go.id/resources/download/pusdatin/pr ofil-kesehatan-indonesia/Profil-Kesehatan-Indonesia2016.pdf. Accessed 11 October 2019. [Report in Indonesian].

31. Ministry of Health Republic of Indonesia (2018) Indonesia health profile $2017 . \quad$ Available: https://pusdatin.kemkes.go.id/resources/download/pusdatin/pr ofil-kesehatan-indonesia/Profil-Kesehatan-Indonesia-tahun2017.pdf. Accessed 11 October 2019. [Report in Indonesian].

32. Ministry of Health Republic of Indonesia (2019) Indonesia health profile 2018. Available: https://pusdatin.kemkes.go.id/resources/download/pusdatin/pr ofil-kesehatan-

indonesia/PROFIL_KESEHATAN_2018_1.pdf. Accessed 11 October 2019. [Report in Indonesian].

33. Purniti PS, Subanada IB, Kari IK, Arhana B, Iswari IS, Tarini NMA (2011) Pneumococcal surveillance and the impact of pneumonia in children under five. Sari Pediatri 12: 359-364. Available: $\quad$ https://saripediatri.org/index.php/saripediatri/article/view/490/427. Accessed 08 October 2019. [Article in Indonesian].

34. National Institute of Health Research and Development Ministry of Health Republic of Indonesia (2019) Basic health research $2018 . \quad$ Available: http://labdata.litbang.kemkes.go.id/images/download/laporan/ RKD/2018/Laporan_Nasional_RKD2018_FINAL.pdf. Accessed 11 October 2019. [Report in Indonesian].

35. Agustiningsih A, Herman R, Ramadhany R, Pratiwi E, Puspa KD, Setiawaty V (2012) Viral and bacterial infection among hospitalized-suspected influenza $\mathrm{A} / \mathrm{H} 5 \mathrm{~N} 1$ patients in Indonesia, 2008-2009. Med J Indones 21.

36. Indawati W, Setyanto DB, Kaswandani N (2014) Influenza A and $\mathrm{B}$ infection among children with influenza like illness (ILI) or pneumonia in Jakarta. Sari Pediatri 16: 136-142. Available: https://saripediatri.org/index.php/sari-

pediatri/article/view/190/50. Accessed 11 October 2019. [Article in Indonesian].

37. Nataprawira HM, Phangkawira E (2015) A retrospective study of acute pertussis in Hasan Sadikin Hospital-Indonesia. J Acute Dis 4: 147-151.

38. Prawira Y, Murniati D, Rusli A, Giriputro S, Setiawaty V, Oswari H, Said M (2012) Clinical, laboratory, and radiologic characteristics of confirmed avian influenza (H5N1). Southeast Asian J Trop Med Public Health 43: 877-889.

39. Sari IP, Nuryastuti T, Wahyono D (2017) The Study of Multidrug-Resistance in Neonatal Intensive Care Unit at the Central Java Hospital. Asian J Pharm Clin Res10.

40. Yuliarti K, Hadinegoro SR, Supriyatno B, Karuniawati A (2012) Invasive pneumococcal disease among hospitalized children aged 28 days to 60 months in Jarkarta. SE Asian Journal Trop Med Public Health 43: 136-144.

41. Widoretno W, Saragih SM, Lokida D (2012) Respiratory viruses mostly found in children under five with SARI (Severe Acute Respiratory Infections). Jurnal Biotek Medisiana Indonesia

1:

93-98.
Available:https://ejournal2.litbang.kemkes.go.id/index.php/jb mi/article/view/1530/836. Accessed 11 October 2019. [Article in Indonesian].

42. Setyati A, Murni IK (2012) Microbial Patterns of Pneumonia Patients in the Pediatric Intensive Care Unit (PICU) Dr. Sardjito Hospital. Media Medika Indonesiana 43(6), 195-200. Available:https://ejournal.undip.ac.id/index.php/mmi/article/v iew/4579. Accessed 11 October 2019. [Article in Indonesian].

43. Gatera VA, Muhtadi A, Halimah E, Prasetyo D (2014) Association of Pattern of Bacteria Sensitivity During the Empirical Antibiotics Use to the Achievement of Clinical Outcome in Pediatric Patients with Pneumonia. Indonesian Journal of Clinical Pharmacy, 3(4), 127-134. Available: http://jurnal.unpad.ac.id/ijcp/article/view/12843/pdf. Accessed 11 October 2019. [Article in Indonesian]

44. Fadlyana E, Dunne EM, Rusmil K, Tarigan R, Sudigdoadi S, Murad C, Watts E, Nguyen C, Satzke C, Dewi NE, Indriyani SAK, Yani FF, Mulholland K, Kartasasmita C (2018) Risk factors associated with nasopharyngeal carriage and density of Streptococcus pneumoniae, Haemophilus influenzae, Moraxella catarrhalis, and Staphylococcus aureus in young children living in Indonesia. Pneumonia 10: 14.

45. Murad C, Dunne EM, Sudigdoadi S, Fadlyana E, Tarigan R, Pell CL, Watts E, Nguyen CD, Satzke C, Hinds J, Dewi MM, Dhamayanti M, Sekarwana N, Rusmil K, Mulholland EK, Kartasasmita C (2019) Pneumococcal carriage, density, and co-colonization dynamics: A longitudinal study in Indonesian infants. Int J Infect Dis 86: 73-81.

46. Hatmaningtyas LLA (2013) Risk factors for colonization of Klebsiella sp in toddler nasopharynx. (Undergraduate). Diponegoro University, Semarang. Available: http://eprints.undip.ac.id/44028/. Accessed 11 October 2019. [Article in Indonesian].

47. Hikmawati (2010) Differences in the colonization pattern of potential bacterial respiratory pathogens in the nasopharynx of children and healthy adults. (Undergraduate). Diponegoro University, Semarang. Available: http://eprints.undip.ac.id/23387/. Accessed 11 October 2019. [Article in Indonesian].

48. Ramachandran P, Nedunchelian K, Vengatesan A, Suresh S (2012) Risk factors for mortality in community acquired pneumonia among children aged 1-59 months admitted in a referral hospital. Indian Pediatr 49: 889-895.

49. Lazzerini M, Seward N, Lufesi N, Banda R, Sinyeka S, Masache G, Nambiar B, Makwenda C, Costello A, McCollum ED, Colbourn T (2016) Mortality and its risk factors in Malawian children admitted to hospital with clinical pneumonia, 2001-12: a retrospective observational study. Lancet Glob Healt 4: e57-68.

50. Jonnalagadda S, Rodriguez O, Estrella B, Sabin LL, Sempertegui F, Hamer DH (2017) Etiology of severe pneumonia in Ecuadorian children. PLoS One 12: e0171687.

51. Jain S, Williams DJ, Arnold SR, Ampofo K, Bramley AM, Reed C, Stockmann C, Anderson EJ, Grijalva CG, Self WH, Zhu Y, Patel A, Hymas W, Chappell JD, Kaufman RA, Kan JH, Dansie D, Lenny N, Hillyard DR, Haynes LM, Levine M, Lindstrom S, Winchell JM, Katz JM, Erdman D, Schneider E, Hicks LA, Wunderink RG, Edwards KM, Pavia AT, McCullers JA, Finelli L (2015) Community-acquired pneumonia requiring hospitalization among U.S. children. N Engl J Med 372: 835-845.

52. Causes of severe pneumonia requiring hospital admission in children without HIV infection from Africa and Asia: the 
PERCH multi-country case-control study (2019) Lancet, 394: 757-779.

53. Ning G, Wang X, Wu D, Yin Z, Li Y, Wang H, Yang W (2017) The etiology of community-acquired pneumonia among children under 5 years of age in mainland China, 2001-2015: A systematic review. Hum Vaccin Immunother 13: 2742-2750.

54. Turner P, Turner C, Jankhot A, Helen N, Lee SJ, Day NP, White NJ, Nosten F, Goldblatt D (2012) A longitudinal study of Streptococcus pneumoniae carriage in a cohort of infants and their mothers on the Thailand-Myanmar border. PLoS One 7 e38271.

55. Jauneikaite E, Jefferies JM, Hibberd ML, Clarke SC (2012) Prevalence of Streptococcus pneumoniae serotypes causing invasive and non-invasive disease in South East Asia: a review. Vaccine 30: 3503-3514.

\section{Corresponding author}

Vincentia Rizke Ciptaningtyas, MD. MSc.

Department of Microbiology, Faculty of Medicine, Universitas Diponegoro

J1. Prof. H. Soedarto, SH, Tembalang, Semarang 50275 Indonesia Tel: +6202476928010

Fax: +62 02476928010

Email: ciptaningtyas_vr@fk.undip.ac.id, Rizke.Ciptaningtyas@radboudumc.nl

Conflict of interests: No conflict of interests is declared. 